\title{
PHILOS plate fixation with polymethyl methacrylate cement augmentation of an osteoporotic proximal humerus fracture
}

\author{
Do-Young Kim, Tae-Yeong Kim, Jung-Taek Hwang \\ Department of Orthopedic Surgery, Chuncheon Sacred Heart Hospital, Hallym University College of Medicine, Chuncheon, Korea
}

PHILOS plate fixation in osteoporotic proximal humerus fracture of old age is well-known for high complication rate, especially metal failure, providing various augmentation techniques, such as calcium phosphate cement, allogenous or autologous bone graft. We report a case of polymethyl methacrylate augmentation to provide appropriate reduction with a significant mechanical support. This can be a treatment option for displaced unstable osteoporotic proximal humerus fracture with marked bony defect.

Keywords: PMMA cement augmentation; Osteoporotic proximal humerus fractures; PHILOS plate fixation

Proximal humerus fractures are common in the elderly, the second most common fracture in the upper extremity, and lead to high morbidity and loss of function [1,2]. The incidence of proximal humerus fractures in Finland was 82 per 100,000 person-years, and $73 \%$ of patients with proximal humerus fractures were women. The Incidence increased with older age groups [3]. Another study revealed that osteoporotic bone increased the risk of proximal humerus fractures and suggested that the high incidence of proximal humerus fractures in older female patients is likely to be related to osteopenia and osteoporosis [4]. Because of the various complications after fixation of a proximal humerus fracture using a plate (e.g., osteonecrosis of the humeral head, humeral head settling with loss of neck-shaft angle, nonunion or malunion, screw penetration into the glenohumeral joint, and implant failure), there has yet to be an accepted consensus on the clear surgical indication for treatment of theses fractures [5].
During surgery for a proximal humerus fracture, a surgeon sometimes encounters a metaphyseal void after reduction of impaction of the metaphysis. Several strategies exist for void filling to help structural stability, enhance healing, and minimize failure of fixation [6]. Bone cement is commonly used in orthopedic surgery due to its osteoconductive properties and resistance to compression force [7]. For example, augmentation with calcium phosphate cement in the treatment of proximal humeral fractures with locked plates increased fracture settling and significantly decreased intra-articular screw penetration in one report [6]. Herein, we present a case of polymethyl methacrylate (PMMA) cement augmentation in a failed osteoporotic proximal humerus fracture.

\section{CASE REPORT}

This study was approved by the Institutional Review Board of 
Hallym University Chuncheon Sacred Heart Hospital (IRB No. 2020-07-003).

A 56-year-old female was sent to our emergency department after a fall in which she landed on her left arm. The patient complained about left shoulder pain, swelling and ecchymosis. On palpation, tenderness was noted all around the shoulder, especially at the level of the axilla. Both neurological and vascular examinations were normal. Anteroposterior X-ray and computed tomography of the left shoulder showed a two-part surgical neck fracture of the proximal humerus (Fig. 1). A preoperative bone mineral density showed osteoporosis $(\mathrm{T}=-3.8)$.

Three days after the injury, an open reduction and internal fixation (ORIF) was performed with the Proximal Humerus Internal Locking System (PHILOS; DePuy Synthes, Raynham, MA, USA) plate and screws. On postoperative day (POD) 4, the patient fell down from her bed, and there was screw back-out and reduction loss at the humeral head (Fig. 2).
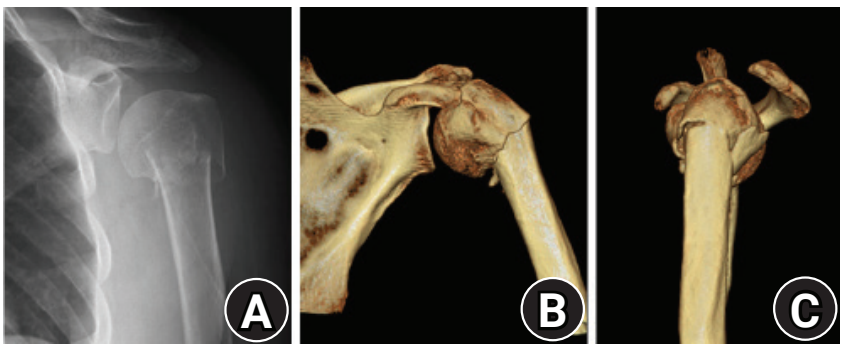

Fig. 1. Preoperative anteroposterior (AP) X-ray and computed tomography showing two-part surgical neck fracture with metaphyseal impaction. (A) Shoulder AP X-ray showed two-part fracture of proximal humerus. (B) Anterior view of three-dimensional computed tomography (3D CT) showed two-part fracture with varus angulation. (C) Lateral view of 3D CT showed two-part fracture.
Thus, revisional ORIF was done on POD 7. Using the previous incision (deltopectoral approach), we removed the previous locking plate. After the revisional ORIF with a PHILOS plate and screws, including a long calcar screw, a metaphyseal defect developed. Thus, we put PMMA cement in the void area to fill the defect and provide structural stability (Fig. 3A, B). We applied an abductor brace. The postoperative X-ray showed firm fixation with the PHILOS plate and cement augmentation (Fig. 3C, D).

After 4 weeks of immobilization, the brace was removed, and the patient started exercise to increase the range of motion of the shoulder joint (forward elevation, $120^{\circ}$; external oration, $60^{\circ}$; internal rotation, L3 level). At that time, a follow-up X-ray showed healing of the fracture without screw backout or loss of reduction. Three months after the revision operation, the patient's range of motion was within the normal range (forward elevation, $150^{\circ}$; external oration, $80^{\circ}$; internal rotation, T8), her pain was tempered
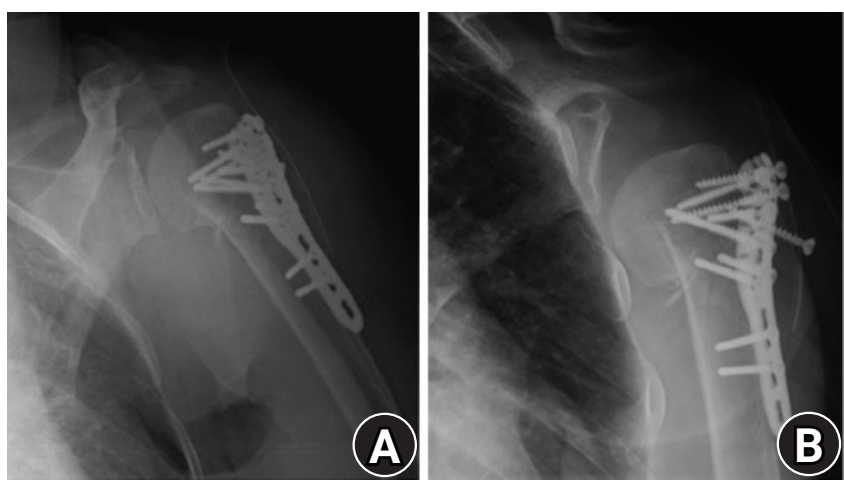

Fig. 2. Open reduction internal fixation with locking plate and screws. (A) Postoperative anteroposterior (AP) X-ray showed reduced state. (B) Metal failure was shown after fall-down on AP X-ray at postoperative day 4 .
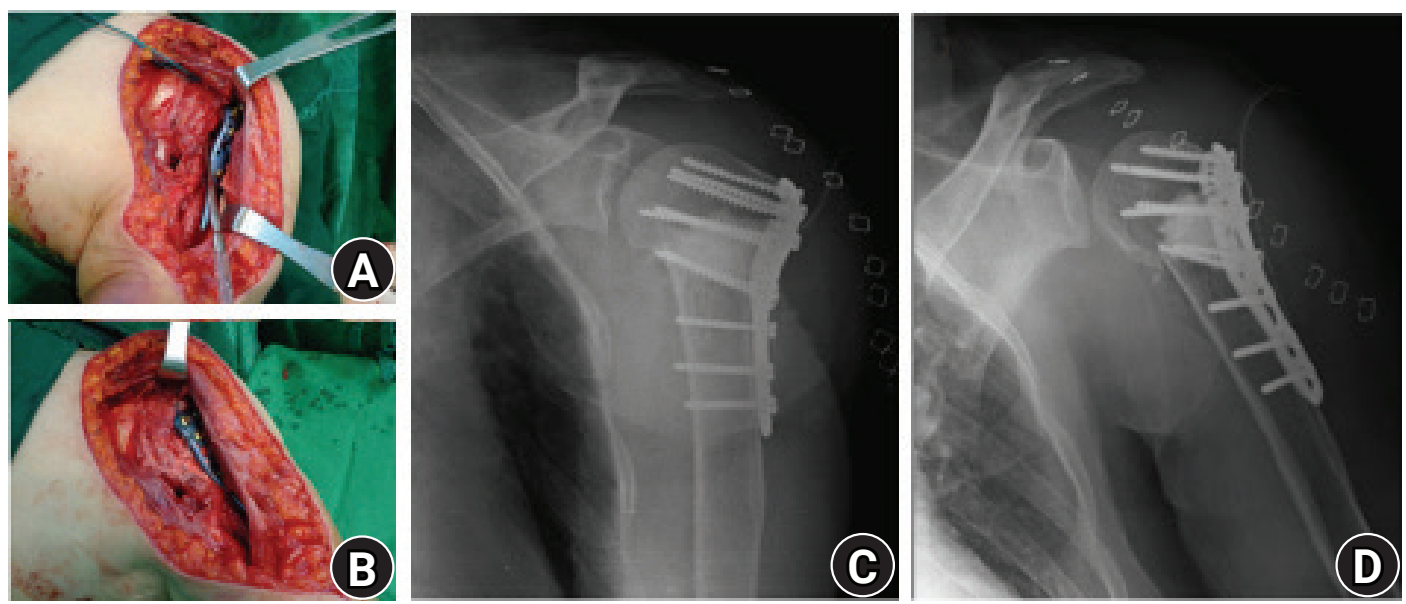

Fig. 3. PolyMethyl methacrylate (PMMA) cement was inserted into the metaphyseal area to fill the void. (A) The metaphysea void was shown. (B) The metaphyseal void was filled with PMMA cement. (C) Shoulder true anteroposterior (AP) X-ray showed PMMA cement was inserted to the metaphyseal void. (D) Open reduction and internal fixation with PMMA cement augmentation showed firm fixation on shoulder AP X-ray. 

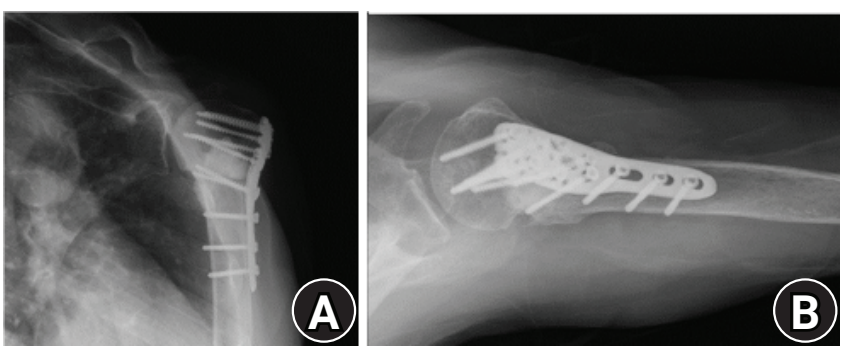

Fig. 4. Healing of fracture without screw back-out or loss of reduction 6 months later. Union is shown with no sign of avascular necrosis. (A) Union was shown on shoulder true anteroposterior X-ray at 6 months after revision surgery. (B) Shoulder axillary X-ray showed union at 6 months after revision surgery.

(visual analog scale score, 1), and the function of her shoulder was improved (American Shoulder and Elbow Surgeons [ASES] score, 73.3; Korean Shoulder Scoring [KSS] system score, 82).

Finally, 6 months after the revision operation, the patient was pain-free with full range movement of the left shoulder and improved clinical outcomes (ASES score, 80.2; KSS score, 92). $\mathrm{X}$-rays revealed union of the proximal humerus fracture with no sign of avascular necrosis of the humeral head (Fig. 4).

\section{DISCUSSION}

The present case demonstrated a satisfactory result from ORIF using a precontoured PHILOS plate combined with PMMA cement augmentation for an osteoporotic proximal humerus fracture. Compared to other materials, such as calcium phosphate cement, allogeneic bone or autologous bone, PMMA cement provides a reduced fracture with significant mechanical support [6-9].

Osteoporotic proximal humerus fracture has high rates of complication and reoperation. There are many augmentation techniques for fixation with a plate as follows: structural allogeneic or autologous bone grafting to enhance the medial support, filling the metaphyseal void with synthetic bone substitutes or bone grafts, and screw-tip augmentation with bone cement [6]. Some studies revealed that bone grafting to fill the void had a positive effect on healing the fracture and reducing the failure rate [8]. One review showed that PMMA augmentation of proximal femoral nail fixation might have the potential to prevent reoperations by strengthening the osteosynthesis construct [9]. In the present case, there is a possibility that some thermal injury occurred from the PMMA cement, but it was used for its ability to provide mechanical support.

Although bone graft or calcium phosphate cement might be better than PMMA cement in the aspect of biocompatibility, PMMA cement augmentation could be better for unstable fractures with severe osteoporotic bone in the aspect of mechanical support. Therefore, the present case report provides another surgical option for displaced osteoporotic proximal humerus fractures with significant bony defects: ORIF with PMMA cement augmentation. Moreover, this option may also be applied for a revisional ORIF of an osteoporotic proximal humerus fracture.

\section{ORCID}

Do-Young Kim

Tae-Yeong Kim

Jung-Taek Hwang https://orcid.org/0000-0003-3735-1640

https://orcid.org/0000-0001-6384-1438

https://orcid.org/0000-0003-4189-084X

\section{REFERENCES}

1. Court-Brown CM, Garg A, McQueen MM. The epidemiology of proximal humeral fractures. Acta Orthop Scand 2001;72:36571.

2. Bell JE, Leung BC, Spratt KF, et al. Trends and variation in incidence, surgical treatment, and repeat surgery of proximal humeral fractures in the elderly. J Bone Joint Surg Am 2011; 93:121-31.

3. Launonen AP, Lepola V, Saranko A, Flinkkilä T, Laitinen M, Mattila VM. Epidemiology of proximal humerus fractures. Arch Osteoporos 2015;10:209.

4. Lee SH, Dargent-Molina P, Bréart G; EPIDOS Group. Epidemiologie de l'Osteoporose Study. Risk factors for fractures of the proximal humerus: results from the EPIDOS prospective study. J Bone Miner Res 2002;17:817-25.

5. Barlow JD, Logli AL, Steinmann SP, et al. Locking plate fixation of proximal humerus fractures in patients older than 60 years continues to be associated with a high complication rate. J Shoulder Elbow Surg 2020;29:1689-94.

6. Egol KA, Sugi MT, Ong CC, Montero N, Davidovitch R, Zuckerman JD. Fracture site augmentation with calcium phosphate cement reduces screw penetration after open reduction-internal fixation of proximal humeral fractures. J Shoulder Elbow Surg 2012;21:741-8.

7. Bajammal SS, Zlowodzki M, Lelwica A, et al. The use of calcium phosphate bone cement in fracture treatment: a meta-analysis of randomized trials. J Bone Joint Surg Am 2008;90:1186-96.

8. Mathison C, Chaudhary R, Beaupre L, Reynolds M, Adeeb S, Bouliane M. Biomechanical analysis of proximal humeral fixation using locking plate fixation with an intramedullary fibular allograft. Clin Biomech (Bristol, Avon) 2010;25:642-6.

9. Kammerlander C, Hem ES, Klopfer T, et al. Cement augmentation of the proximal femoral nail antirotation (PFNA): a multicentre randomized controlled trial. Injury 2018;49:1436-44. 